\title{
MiR-15b Targets Cyclin D1 to Regulate Proliferation and Apoptosis in Glioma Cells
}

\author{
Guan Sun, ${ }^{1}$ Lei Shi, ${ }^{2}$ Shushan Yan, ${ }^{3}$ Zhengqiang Wan, ${ }^{1}$ Nan Jiang, ${ }^{1}$ Linshan Fu, ${ }^{1}$ \\ Min $\mathrm{Li}^{4}{ }^{4}$ and Jun Guo ${ }^{1}$ \\ ${ }^{1}$ Department of Neurosurgery, Fourth Affiliated Hospital of Nantong University, First Hospital of Yancheng, Yancheng 224001, China \\ ${ }^{2}$ Department of Neurosurgery, The First People's Hospital of Kunshan Affiliated with Jiangsu University, Suzhou 215300, China \\ ${ }^{3}$ Department of Medical Oncology, The Eighty-First Hospital of People's Liberation Army, Nanjing 210002, China \\ ${ }^{4}$ Department of Neurosurgery, Jiangning Hospital Affiliated with Nanjing Medical University, Nanjing 211100, China
}

Correspondence should be addressed to Min Li; sbn133@163.com and Jun Guo; junguo8916@163.com

Received 12 April 2014; Revised 8 May 2014; Accepted 12 May 2014; Published 4 June 2014

Academic Editor: Eiichi Ishikawa

Copyright (C) 2014 Guan Sun et al. This is an open access article distributed under the Creative Commons Attribution License, which permits unrestricted use, distribution, and reproduction in any medium, provided the original work is properly cited.

\begin{abstract}
Aim. To investigate the role and mechanism of miR-15b in the proliferation and apoptosis of glioma. Methods. The miR-15b mimics were transfected into human glioma cells to upregulate the miR-15b expression. Cyclin D1 was determined by both western blotting analysis and luciferase reporter assay. Methylthiazol tetrazolium (MTT) and flow cytometry were employed to detect the cell proliferation, cell cycle, and apoptosis. Results. Overexpression of miR-15b inhibits proliferation by arrested cell cycle progression and induces apoptosis, possibly by directly targeting Cyclin D1. Both luciferase assay and bioinformatics search revealed a putative target site of miR-15b binding to the $3^{\prime}$-UTR of Cyclin D1. Moreover, expression of miR-15b in glioma tissues was found to be inversely correlated with Cyclin D1 expression. Enforced Cyclin D1 could abrogate the miR-15b-mediated cell cycle arrest and apoptosis. Conclusions. Our findings identified that miR-15b may function as a glioma suppressor by targeting the Cyclin D1, which may provide a novel therapeutic strategy for treatment of glioma.
\end{abstract}

\section{Background}

Glioma represents the majority of primary malignant brain tumors with low survival rate [1]. Despite advance in surgery, radiotherapy, and chemotherapy, over $50 \%$ of patients die within one year by diagnosis of glioblastoma [2]. Thus, it is very urgent to explore the mechanisms that underlie the occurrence and development of glioma.

In the past few years, miRNAs considered as glioma biological targets were widely studied. To our knowledge, a growing body of evidence indicates that miRNAs play fundamental roles in the tumorigenesis of human cancer including glioma, affecting cell survival, proliferation, differentiation, metabolism, angiogenesis, and stem cell generation $[3,4]$. More and more numbers of miRNAs including miR21, miR-125b, let-7, miR-9, and miR-137, as tumor suppressors or oncogenes by negatively regulating oncogenes or tumorsuppressor genes, have been verified to expose aberrant expression in glioma [5-9]. One given miRNA is supposed to have multiple gene targets while one given target also may have simultaneous multiple upstream miRNAs.

For example, we have recently reported that forced expression of miR-137 suppresses proliferation and invasiveness in cultured glioma cells by directly targeting Racl. Till now, it has been verified that miR-137 directly regulates a wide variety of genes such as CDC42, CDK6, KIT, and AEG-1 in several cancer tissues [10-12]. MiR-34a negatively regulates surviving protein expression and inhibits gastric cancer cell proliferation and invasion [13]. Thus, altered expression miRNAs and their simultaneous target genes have been demonstrated to contribute to the development of various cancers.

Recently, miR-15b has been declared to be involved in progression of an element of cancer tissues. Ectopic miR-15b induces apoptosis by targeting BCL2, sensitized SGC7901/VCR cells to VCR-induced apoptosis [14]. MiR-15b 
directly targeted FUT2 and then increased levels of Globo $\mathrm{H}$ to enhance HCC cell proliferation [15]. However, the molecular biological effects and regulatory mechanism of miR-15b in glioma cells remain elusive.

Here, we investigated the effects of miR-15b in glioma cells and explored the detailed mechanisms involved. Our data revealed that miR-15b was substantially decreased in glioma specimens compared to the matched normal brain tissues. Further studies showed that Cyclin D1 was a novel direct target of miR-15b and was frequently downregulated in glioma tissues and cell lines. MiR-15b inhibited U87 and LN229 glioma cells growth at least partially by regulating the $3^{\prime}$-untranslated regions $\left(3^{\prime}\right.$-UTR) of Cyclin D1. In consequence, miR-15b may act as the role of tumor suppressor in glioma cells.

\section{Materials and Methods}

2.1. Human Tissue Samples and Cell Lines. Human U87 and LN229 glioma cell lines were purchased from the Chinese Academy of Sciences Cell Bank. All cells were maintained in a $37^{\circ} \mathrm{C}, 5 \% \mathrm{CO}_{2}$ incubator in Dulbecco's modified eagle's medium (DMEM) (Gibco, USA) supplemented with 10\% fetal bovine serum (Invitrogen).

2.2. Oligonucleotides and Cell Transfection. The oligonucleotides were chemically synthesized by GenePharma (Shanghai, China) bases with the following sequences: hsamiR-15b mimics (sense: $5^{\prime}$-UAGCAGCACAUCAUGGUUUACA-3', antisense: $5^{\prime}$-UAAACAUGAUGUGCUGCUGUU- $3^{\prime}$ ) and scrambled miR-15b (sense: $5^{\prime}$-UUCUCCGAACGUGUCACGUTT-3', anti-sense: $5^{\prime}$-ACGUGACACGUUCGGAGAATT- $3^{\prime}$ ). For the Cyclin D1 plasmid vectors (Genesil, Wuhan, China) were constructed by Wuhan Genesil. Vectors were transfected into human glioma cell lines with FuGENE HD6 (Roche, Basel, Switzerland) according to the manufacturer's instructions and screened by the aminoglycoside G418. For transfection, $2 \times 10^{5}$ cells were placed into each well of six well plates for 12 hours. Mimics were allowed to form transfection complexes with Lipofectamine 2000 (Invitrogen) according to the manufacturer's instructions (Invitrogen, Carlsbad, USA), subsequently added to U87 and U229 glioma cells, and left to incubate for $8 \mathrm{~h}$ before medium change. Experiments were divided into three groups as blank control group (blank), miRNA scrambled group (scramble), and hsa-miR-15b mimics group (miR-15b mimics).

2.3. Quantitative Real-Time PCR ( $q R T-P C R)$ Analysis of MiR$15 b$ and Cyclin D1. Total RNA was extracted from clinical tissues and transfected cells, using Trizol reagent (Invitrogen, USA). The ABI 7300 HT sequence detection system (Applied Biosystems, Foster City, CA) was used for Taqman-based real-time reverse transcription-polymerase chain reaction (RT-PCR) assays to detect the relative levels of miR-15b and Cyclin D1 in glioma samples and transfected cells. Primers and probes of the miR-15b and Cyclin D1 for Taqman miRNA assays were purchased from Applied Biosystems.
The quantitative miR-15b and Cyclin D1 expression date was calculated by using a $2^{-} \Delta \Delta$ Ct method.

2.4. MTT Assay. Cells in the log phase of growth were seeded into 96 -well plates at $3 \times 10^{3}$ cells per well. Subsequently, $50 \mu \mathrm{L}$ of MTT dilution ( $5 \mathrm{mg} / \mathrm{mL}$, KeyGEN, China) was added into each well at each day of the consecutive 3 days after transfection and the cells were incubated at $37^{\circ} \mathrm{C}$ for additional $4 \mathrm{~h}$. The supernatant was discarded and $200 \mu \mathrm{L}$ of DMSO was added to each well to dissolve the precipitate. Optical density (OD) was measured at the wavelength of $570 \mathrm{~nm}$ and data were presented as the mean \pm SD.

2.5. Cell Cycle Assay. Cells were washed with PBS and fixed with $70 \%$ ethanol for at least $1 \mathrm{~h}$. After extensive washing, the cells were suspended in HBSS (Hank's balanced salt solution) containing $50 \mu \mathrm{g} / \mathrm{mL}$ PI and $50 \mu \mathrm{g} / \mathrm{mL}$ RNase A and incubated for $1 \mathrm{~h}$ at room temperature and analyzed by FACScan (Becton Dickinson, USA). Cell cycle analysis was analyzed by ModFit software. Experiments were performed in triplicate. Results were presented as \% of cell in a particular phase.

2.6. Apoptosis Assay. $1 \times 10^{6}$ cells were plated into 6-well plates, and, after transfection with each oligonucleotide for $48 \mathrm{~h}$, the Annexin V FITC and PI double stain were used to detect and quantify apoptosis by flow cytometry.

2.7. Luciferase Reporter Assay. The full length $3^{\prime}$ UTR region of the Cyclin D1 gene was subcloned into luciferase reporter vectors from human genomic DNA and the mutant Cyclin D1 vectors were constructed. Cells of $60-70 \%$ confluence in 24-well plates were cotransfected with luciferase reporter vectors and miR-15b expressing vectors, and a 1ng pRLSV40 Renilla luciferase construct was used for normalization. After $48 \mathrm{~h}$, luciferase activity was analyzed by the dual-luciferase reporter assay system according to the manufacturer's protocols (Promega, Madison, USA).

2.8. Western Blotting Analysis. At $48 \mathrm{~h}$ after transfection with miR-15b mimics or scramble oligonucleotides, total proteins from control and transfected cells were extracted and the protein concentration was determined by BSA method (keyGEN, China). $30 \mu \mathrm{g}$ of protein lysates was subjected to SDS-PAGE in $10 \%$ acrylamide gel each sample. Then, the electrophoresed proteins were transferred to NC membranes (Millipore Corporation, USA). After that, the membrane was blocked in 5\% nonfat milk and incubated with diluted antibodies against Cyclin D1 (1:200, Santa Cruz, USA) overnight at $4^{\circ} \mathrm{C}$, followed by incubation with HRP-conjugated secondary antibody (1:2500, Santa Cruz, USA). After washing with stripping buffer, the membrane was reprobed with GAPDH (1:5000, Kangchen, China), using ultra enhanced chemiluminescence western blotting detection reagents. All Western bands were quantified by densitometry and are presented in the form of a bar graph. 


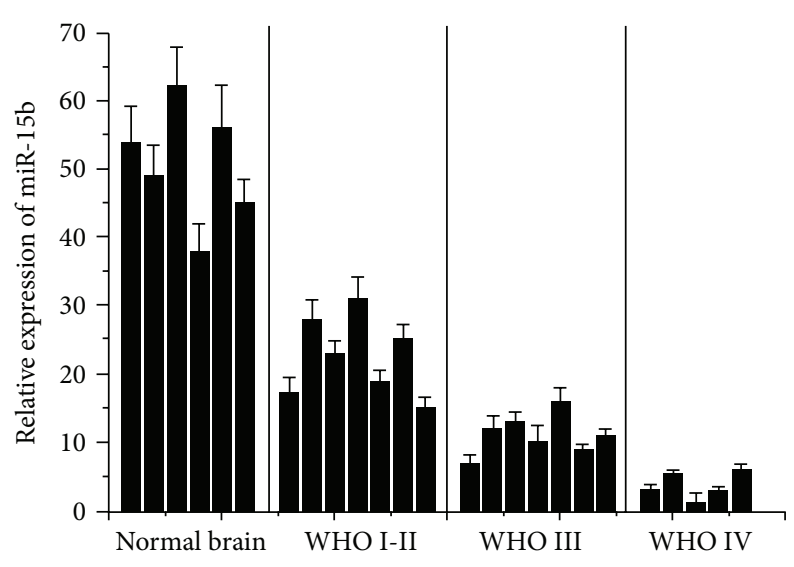

(a)

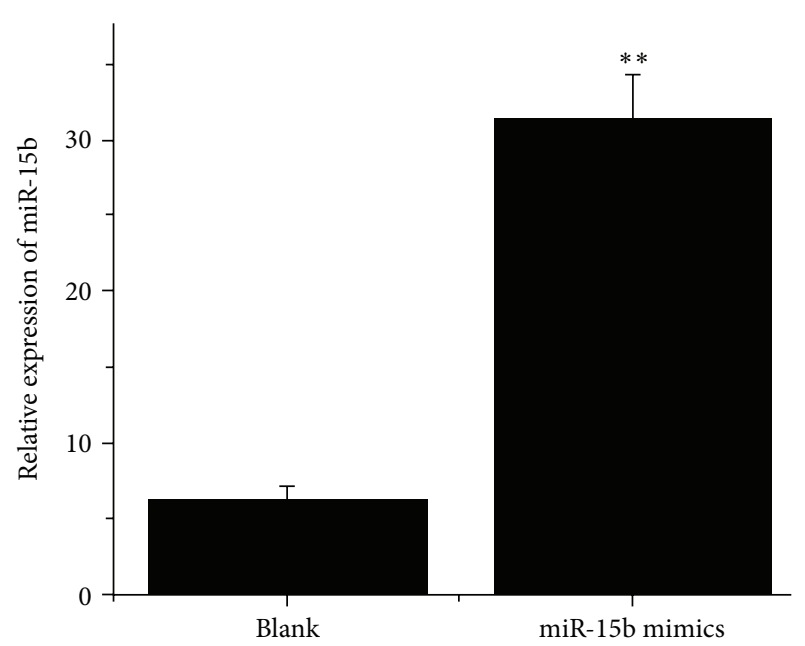

U87

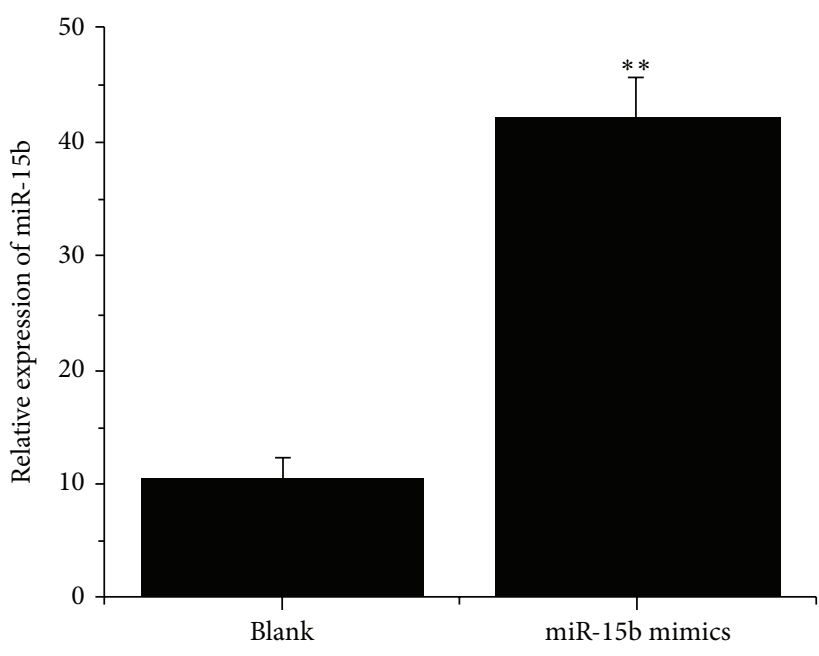

LN229

(b)

(c)

FIGURE 1: (a) MiR-15b expression in glioma tissues and normal brain specimens. The relative levels of miR-15b were measured by real-time PCR assay. (b, c) MiR-15b expression increased about 5.02-fold and 3.98-fold, at $48 \mathrm{~h}$ after transfection of miR-15b mimics in U87 and LN229 cells, respectively. Total RNA was extracted using Trizol reagent. The relative expression of miR-15b was calculated by using a $2^{-} \Delta \Delta \mathrm{Ct}$ method. The data are presented as the mean \pm SD. ${ }^{* *} P<0.05$ compared to the control.

2.9. Statistics Analysis. Data were analyzed with SPSS 13.0 statistical evaluation for data analysis that was determined by $t$ test, one-way ANOVA, and Pearson's correlation analysis. Differences with $P<0.05$ were considered statistically significant.

\section{Results}

3.1. MiR-15b Expression in Glioma Tissues. To explore the expression of miR-15b in glioma tissues, 7 I-II, 7 III, and 5 IV grades and 6 normal brain tissues were examined by realtime PCR assay. As shown in Figure 1, miR-15b expression decreased markedly in glioma specimens in comparison to the normal brain tissues and showed an obviously downward trend with ascending tumor pathological grades (Figure 1(a)). Similarly, lower expression of miR-15b was observed in U87 and L229 glioma cells. To determine the biological functions of miR-15b in glioma cells, we used chemically synthesized modified oligonucleotides to transfect into U87 and L229 cell lines. The results of real-time PCR assay suggested that miR$15 \mathrm{~b}$ was elevated effectively after transfection in glioma cell lines (Figures 1(b) and 1(c)).

3.2. Cyclin D1 Is a Direct Target of MiR-15b. Three bioinformatic algorithms (TargetScan, PicTar, and miRanda) were employed to identify a large number of potential target genes of miR-15b. Among these candidates, Cyclin D1 was selected for further analysis. Binding sites of miR-15b were observed 


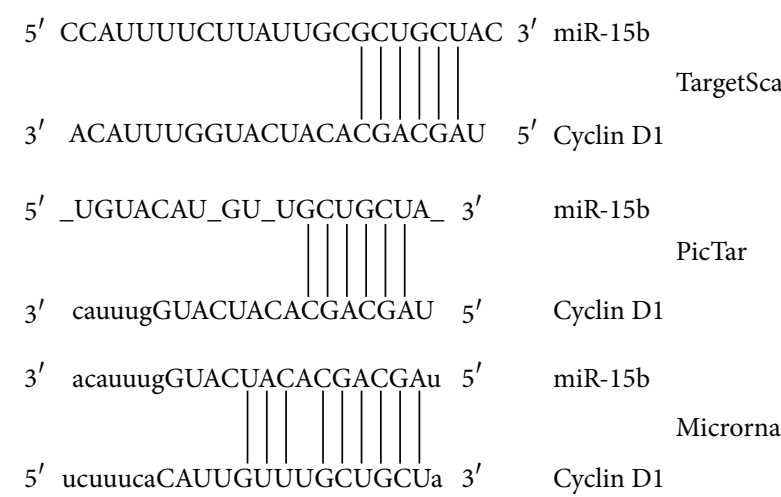

(a)

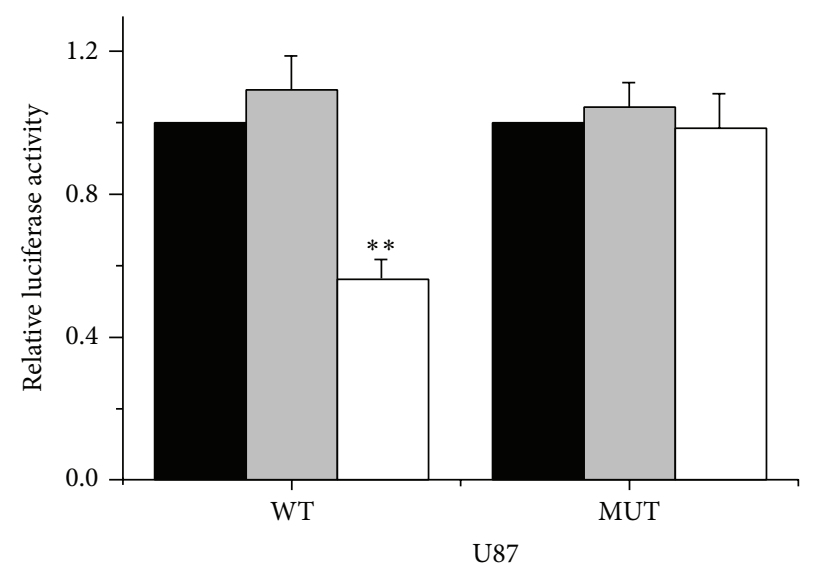

Blank
Scramble $\square$ miR-15b mimics

(c)

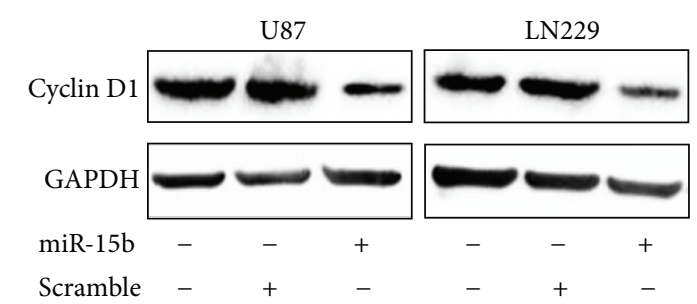

(b)

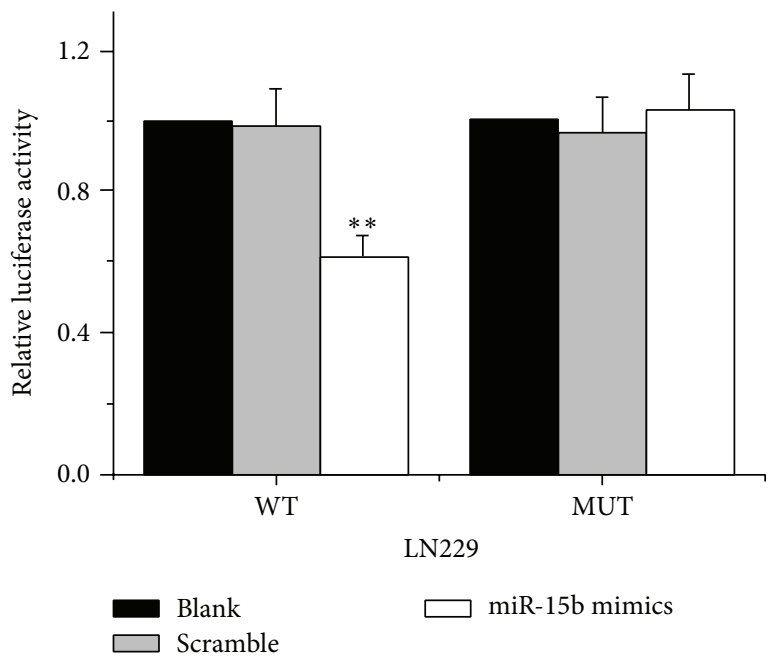

(d)

FIGURE 2: Cyclin D1 is a direct target of miR-15b in glioma cells. (a) Bioinformatics analysis of the predicted interactions of miR-15b with the binding sequence at the $3^{\prime}$ UTR of Cyclin D1 mRNA. (b) Overexpression of miR15b downregulates endogenous Cyclin D1 expression level in U87 and LN229 glioma cells. (c, d) Luciferase assay showed a reduction of luciferase expression following the cotransfection of pGL3-Cyclin $\mathrm{D} 1$ vector together with miR-15b. The data were normalized by the ratio of firefly and Renilla luciferase activities. The data are presented as the mean \pm SD. ${ }^{* *} P<0.05$ compared to the control.

in the $3^{\prime}$-UTR of Cyclin D1 mRNA; we hypothesized that Cyclin D1 may be a direct target of miR-15b (Figure 2(a)). Western blotting analysis showed that miR-15b could reduce the expression of Cyclin D1 in both U87 and LN229 cells (Figure 2(b)). To further confirm whether Cyclin D1 is a direct target of miR-15b, a reporter plasmid harboring the wild-type $3^{\prime}$-UTR region of Cyclin D1 downstream of the luciferase coding region was constructed. The assay denoted that the overexpression of miR-15b induced an obvious decrease in the luciferase activity of the pGL3-WT Cyclin D1 in both U87 and LN229 cells, indicating that miR-15b directly regulates Cyclin D1 gene by binding to $3^{\prime}$ UTR region (Figures 2 (c) and 2(d)). These findings suggested that miR-15b directly regulates Cyclin D1 via binding the $3^{\prime}$-UTR of Cyclin D1.

3.3. Negative Link between MiR-15b and Cyclin D1 Expression in Glioma Tissues. To investigate the association between miR-15b and Cyclin D1 expression in glioma, we analyzed
Cyclin D1 expression by real-time PCR. The higher expression of Cyclin D1 was found in glioma tissues compared to the normal brain tissues. In addition, Pearson's correlation coefficient showed a significant inverse correlation between miR-15b and Cyclin D1 in glioma tissues $(R=-0.79125$ $P<0.01$ ) (Figure 3(b)). These results indicate a negative link miR-15b and Cyclin D1 and further confirm that Cyclin D1 is a direct target of miR-15b.

3.4. MiR-15b Suppresses the Proliferation of U87 and L229 Glioma Cells In Vitro. To determine the effects of miR-15b on proliferation of glioma cells, MTT assay was employed to evaluate the cells growth viability. MiR-15b treated U87 cells showed a significant decrease in proliferation relative to both blank and scramble-treated groups. About $71.17 \pm 6.15 \%$, $52.63 \pm 4.18 \%$, and $49.49 \pm 5.24 \%$ survival rates in $1 \mathrm{~d}, 2 \mathrm{~d}$, and $3 \mathrm{~d}$ after transfected time point were shown, respectively, and the similar inhibitory effects were found in LN229 cell 


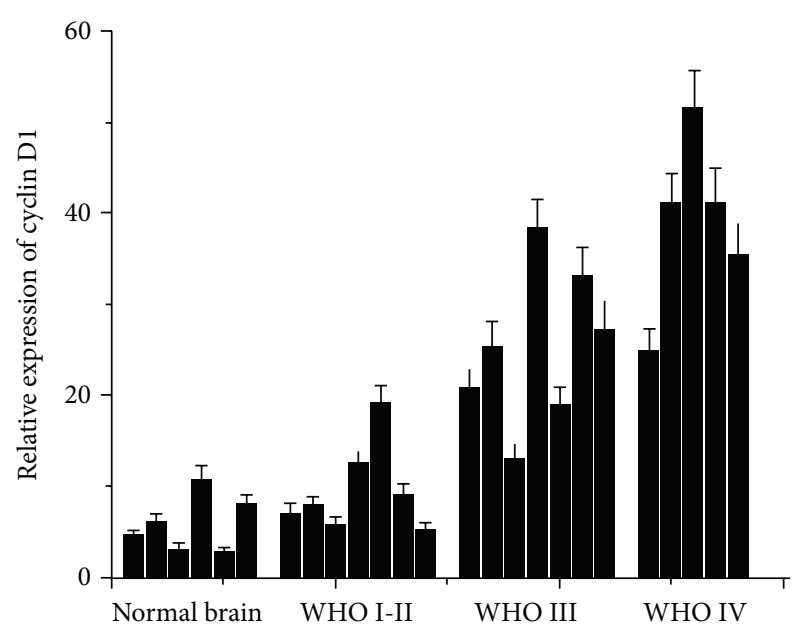

(a)

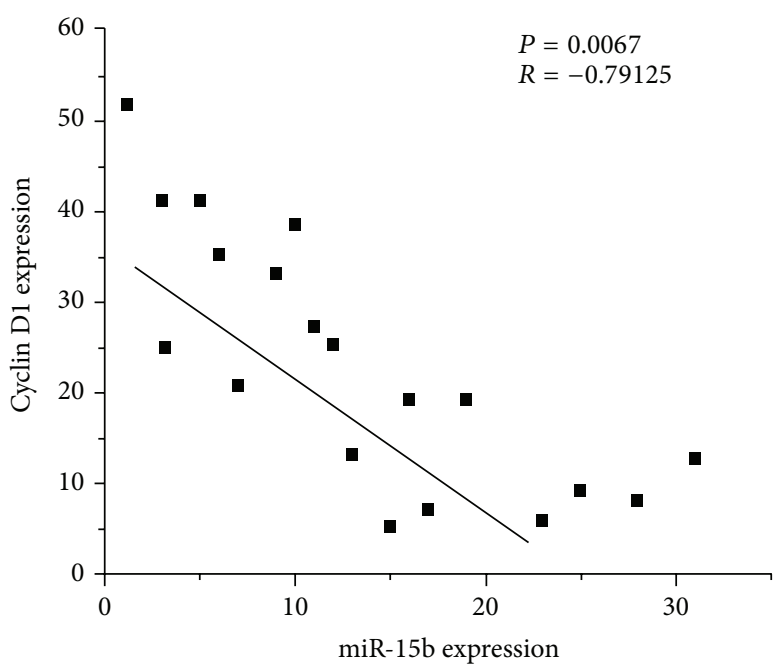

(b)

Figure 3: Negative link between miR-15b and Cyclin D1 expression in glioma tissues. (a) The relative expression of Cyclin D1 was measured by real-time PCR assay. (b) Inverse correlation of miR-15b expression with Cyclin D1 mRNA expression using Pearson's correlation analysis.

(Figure 4(a)). The assay revealed that ectopic expression of miR-15b significantly suppressed the proliferation of glioma cells.

3.5. MiR-15b Results in an Increase of Cell Populations in G0/G1 Phase. The cell cycle distribution by flow cytometry assay was employed to explore why miR-15b inhibits glioma cells. As showed in Figure 4(b), the G1/G0 phase fraction of the control and scramble groups was $54.42 \%$ and $52.72 \%$ while the miR-15b mimics group increased to $69.13 \%$ in U87 cells. In the meanwhile, the G1/G0 phase fraction of the control and scramble groups was 51.84 and $49.56 \%$ while the miR-15b mimics group increased to $66.32 \%$ in LN229 cells. These data suggest that miR-15b mimics lead to the arrest of the cells at G1/G0 phases and delay the progression of cell cycle.

3.6. MiR-15b Induced Apoptosis in U87 and L229 Glioma Cells. To investigate the effects of miR-15b on glioma cell apoptosis, we used miR-15b mimics to transfect into U87 and LN229 glioma cells. At $48 \mathrm{~h}$ after transfection, apoptosis was measured by flow cytometry. The higher apoptotic rates were found among miR-15b mimics treated U87 cells (18.42 \pm 1.46 ) in comparison to the untreated cells. MiR-15b mimics treated LN229 cells also showed considerable apoptotic cells compared to the untreated cells (Figure 4(c)). The assay implied that miR-15b could induce apoptosis in U87 and L229 glioma cells.

3.7. Overexpression of Cyclin D1 Partly Represses the MiR$15 b$ Induced Cell Cycle Arrest in Glioma Cells. To explore the importance of Cyclin D1 in the process of miR-15b-mediated cell cycle and apoptosis, the plasmid mediated overexpression of Cyclin D1 was transfected into glioma cells accompanying miR-15b mimics. As showed in Figure 5(a), western bolt assay significantly showed that enforced expression of Cyclin D1 at least partly repressed the miR-15b-mediated Cyclin D1 expression in both U87 and LN229 cells. Furthermore, ectopic expression of Cyclin D1 statistically counteracted the G1 arrest induced by miR-15b in glioma cells (Figure 5(b)). Likewise, the miR15b-induced cell apoptosis was rescued considerably. Taken together, the results of this rescue experiment demonstrate that reintroduction of Cyclin D1 could abrogate the inhibitory effects of miR-15b-mediated cell cycle arrest and apoptosis, suggesting that Cyclin D1 is a major target of miR-15b involved in the malignant progression of miR-15b.

\section{Discussion}

MiRNAs are a novel class of small nonprotein coding singlestranded RNA molecules, which are essentially posttranscriptional regulators of gene expression. Increasing studies in recent years have confirmed that miRNAs could be involved in a wide range of biological functions in various human cancers. MiRNAs may function as oncogenes or antioncogenes, leading to aberrant cell proliferation and survival by regulating downstream target genes relevant to signal cascades. Thus, it is important to depict the function and mechanism of miRNAs involved in the occurrence and development of glioma.

MiR-15b was firstly reported by Xi et al. The panel found that it was significantly overexpressed in colorectal cancer compared to normal colorectal sample [16]. Wang et al. revealed that miR-15b was upregulated by using miRNA array analyses for age-matched normal cervix and cervical cancer tissues in combination with northern blot verification [17]. In contrast, a number of studies have shown that miR15b statistically reduced in tumor tissues, such as gastric cancer and hepatocellular carcinoma $[14,18,19]$. From above 

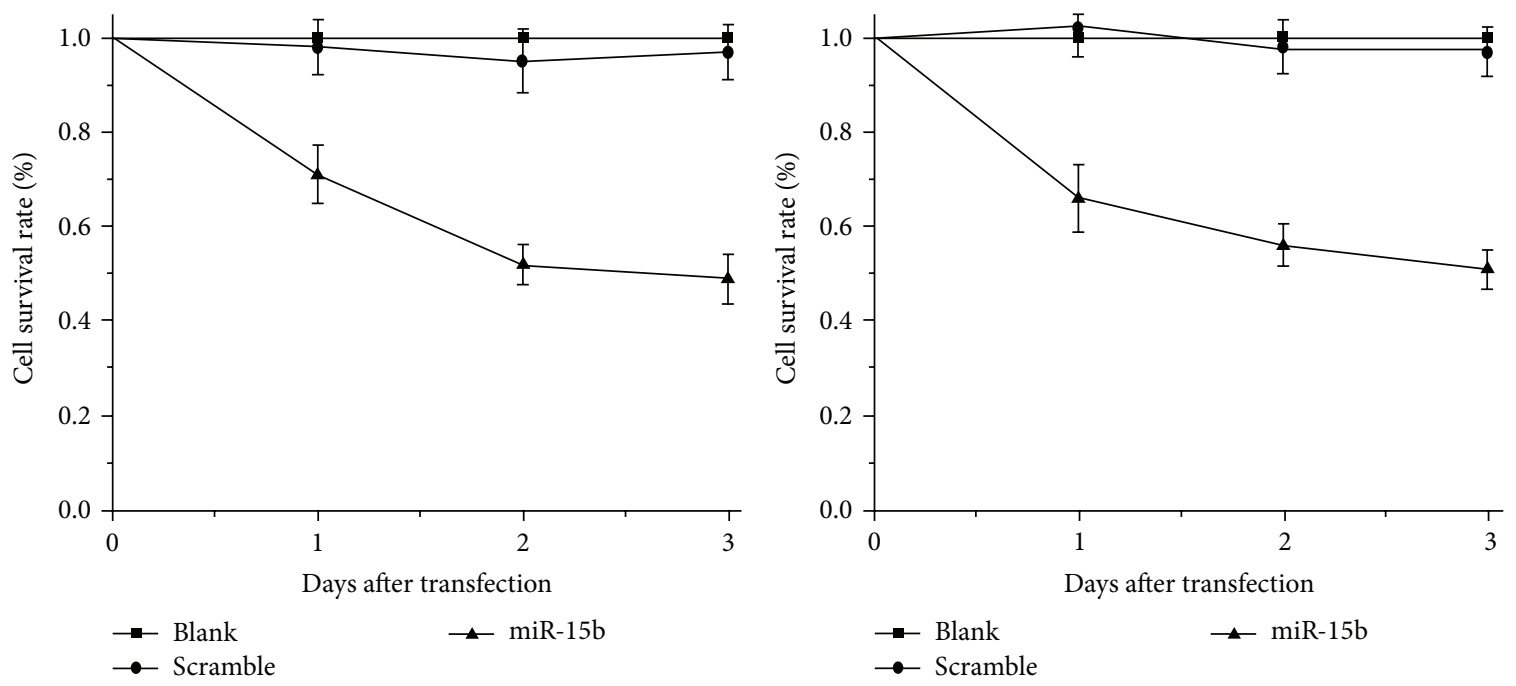

(a)
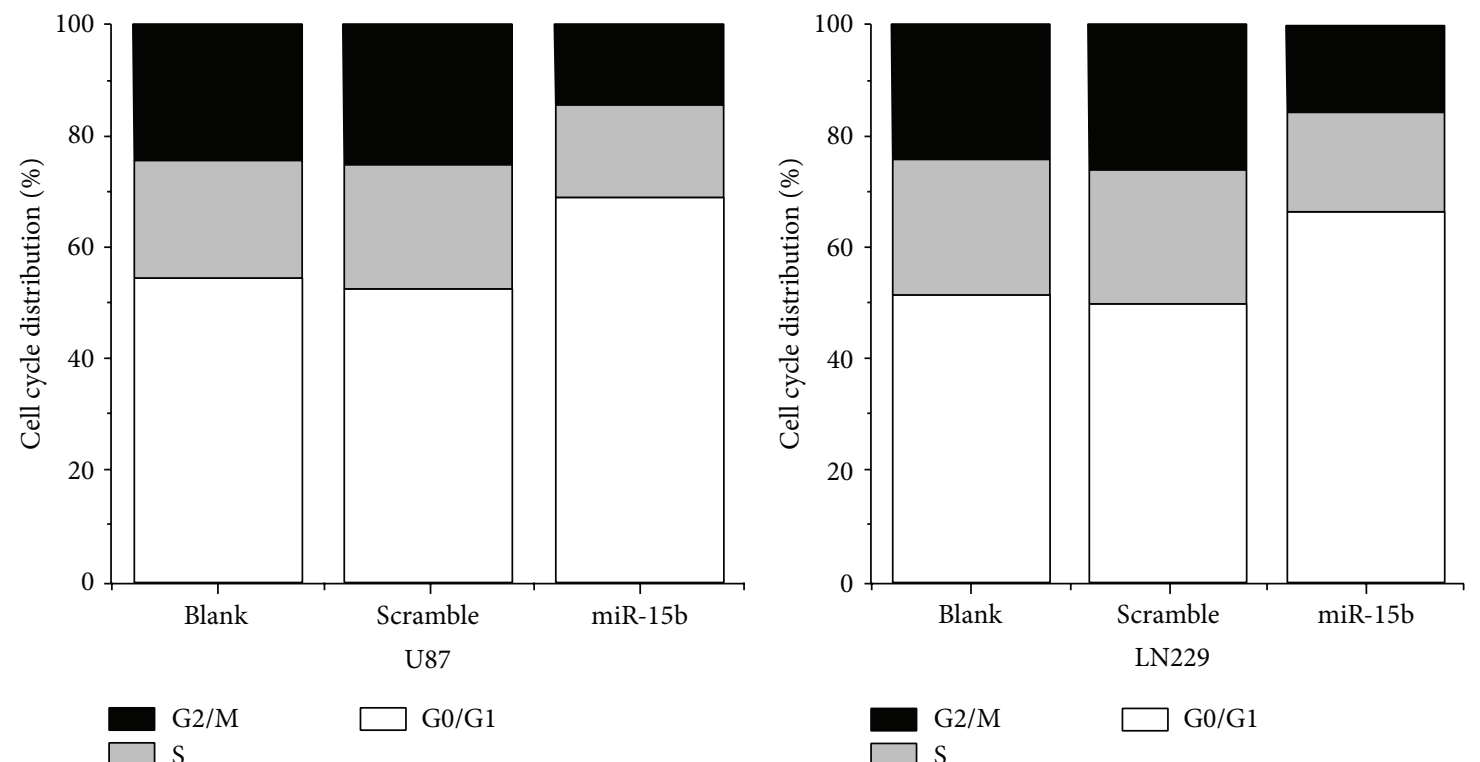

(b)
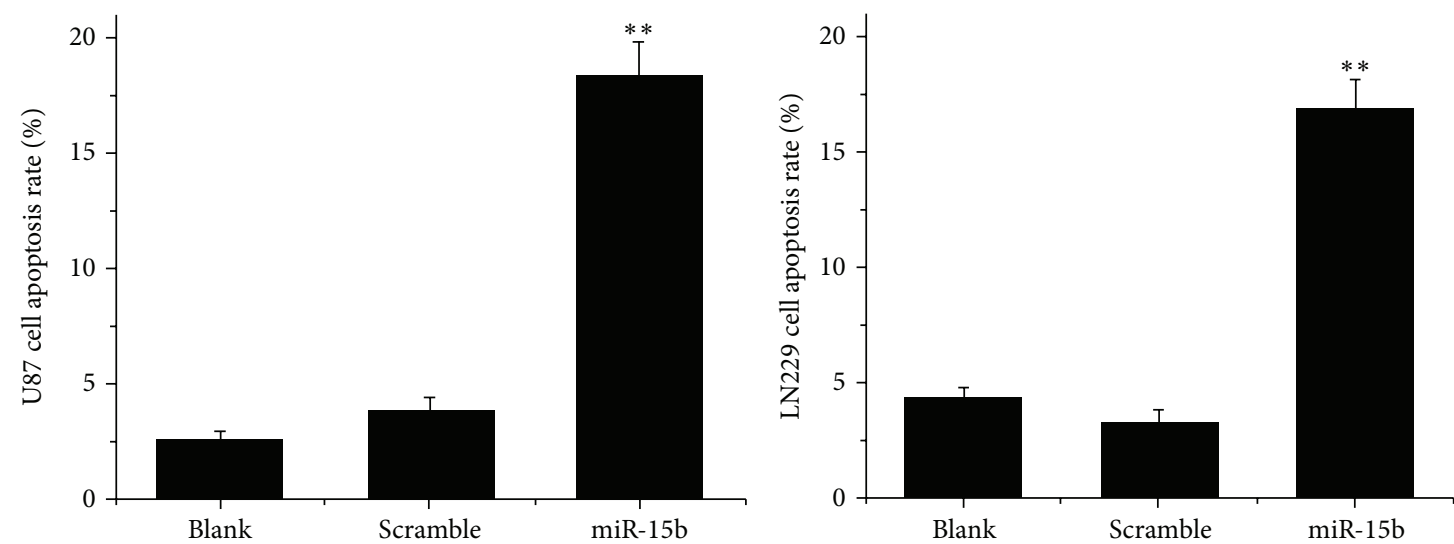

(c)

FIGURE 4: MiR-15b suppresses the growth of glioma cells. (a) MTT assay reveals a significantly inhibitory effect of miR-15b mimics treated cell (Student's $t$-test). (b) Overexpression of miR-15b results in the cell cycle arrest at G0/G1 phases in glioma cells. (c) MiR-15b induced apoptosis in both U87 and L229 glioma cells (Student's $t$-test). The data are presented as the mean \pm SD. ${ }^{* *} P<0.05$ compared to the control. 


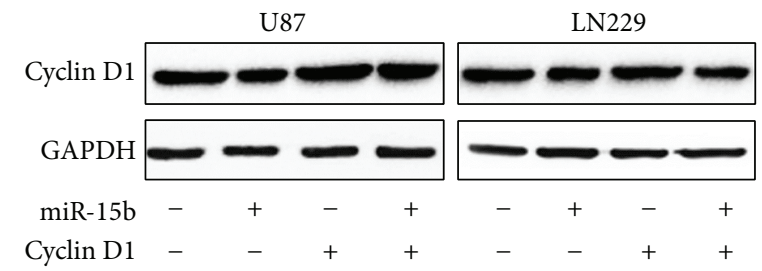

(a)
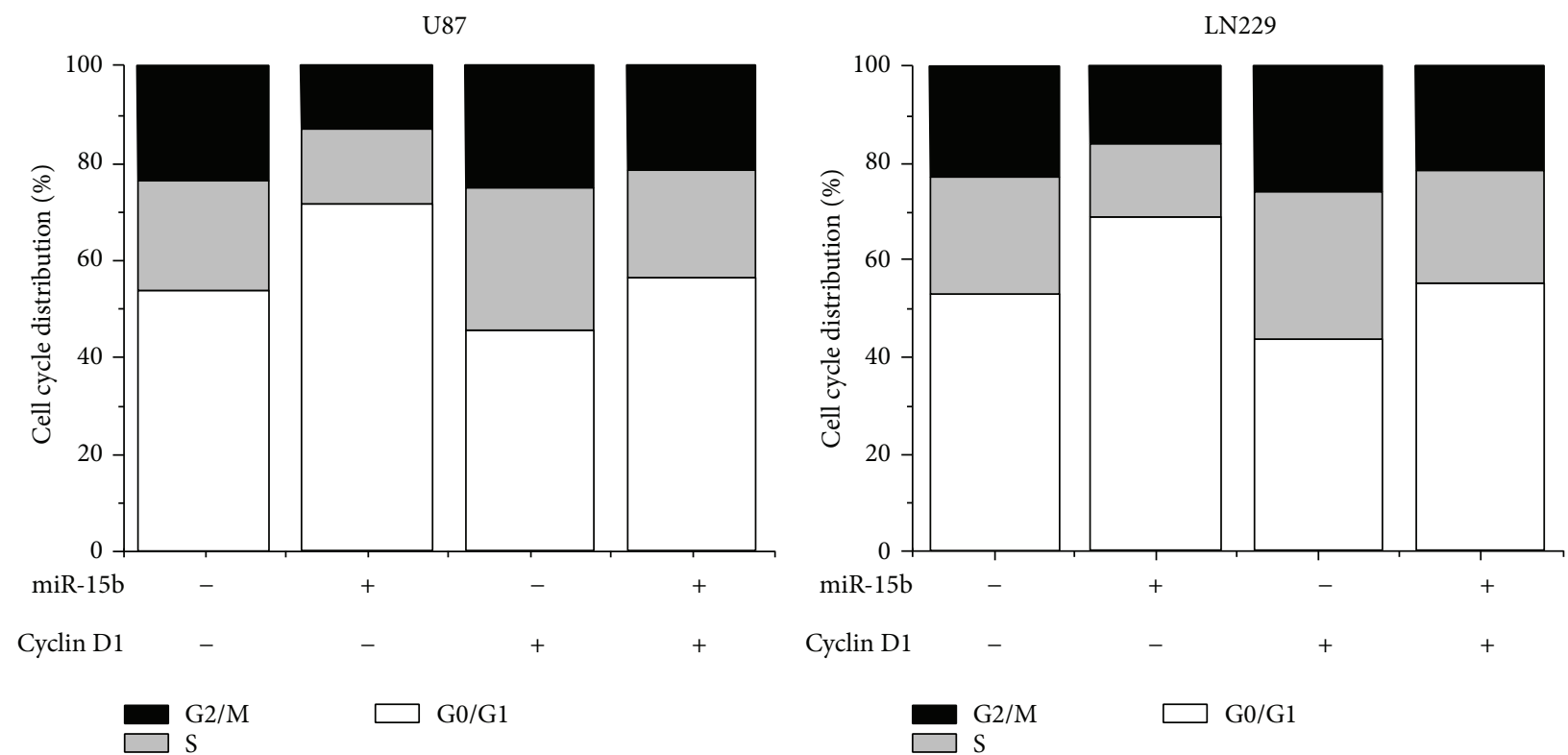

(b)

FIGURE 5: Cyclin D1 plays a crucial role in the suppressive proliferative process of miR-15b in glioma cells. (a) Western bolt analysis displays that ectopic of Cyclin D1 abrogates the miR-15b-mediated Cyclin D1 expression partly. (b) Ectopic expression of Cyclin D1 counteracts the G1 arrest induced by miR-15b in glioma cells. The representative results out of three times of experiments were shown here.

discussion, miR-15b expression was different from diverse organic tissues. Thus, we measured the levels of miR-15b in 19 glioma tissues and 6 normal brain tissues. Our data showed that miR-15b was frequently downregulated in glioma tissue as well as glioma cell lines. Furthermore, miR-15b expression was markedly decreased with ascending glioma malignancy, suggesting that miR-15b exists as an antitumor factor in glioma. Although there have been abundant studies reporting that miR-15b may serve as an essential role in the development and progression of human tumors, the effect of miR-15b in mediating glioma cell growth remains unexplored. To explore the potential molecular mechanism of miR-15b functions in glioma cell lines, we transfected miR15b into U87 and LN229 cells and found that overexpression of miR-15b in vitro statistically inhibited the proliferation of U87 and LN229 glioma cells, arrested miR-15b mimics treated cells in G0/G1 phase, and induced apoptosis in glioma cells.

Cyclin D1 is one of the crucial mitogen cell cycle regulators, which play a pivotal role in the transition of cell cycle from $\mathrm{G} 1$ to $\mathrm{S}$ phase by binding its partners cyclin dependent kinase $4 / 6$ to form an active protein kinase $[20,21]$. Cyclin D1 is frequently overexpressed in a large number of cancers, including colorectal, gastric cancer, nonsmall cell lung cancer, and glioma by diverse mechanisms such as genomic amplification, chromosomal translocations, disruption of normal intercellular trafficking, and proteolysis [2225]. It is generally recognized that Cyclin D1 is associated with tumor malignancy and poor prognosis. Recently, suppression of Cyclin D1 reduced the proliferation and invasiveness of glioma cells while inducing apoptosis, suggesting the crucial role of Cyclin D1 in gliogenesis and defining Cyclin D1 as a promising molecular target for anticancer therapy [26]. In addition, an increasing number of reports have shown that Cyclin D1 was involved in the miRNAs and downstream target genes regulatory networks. MiR-9 suppresses the expression of Cyclin D1 via directly targeting $3^{\prime}$-UTR of Cyclin D1, inhibiting the proliferation, invasion, and metastasis of gastric cancer cell in vitro and in vivo [27]. Abnormal suppression of miR-503 leads to the increase in the Cyclin D1 level, which may promote carcinogenesis in endometrioid endometrial cancer [28]. miR-195 inhibits glioma cell proliferation by downregulating expression of Cyclin D1 and cyclin E1, through directly targeting the $3^{\prime}$ UTR of Cyclin D1 and cyclin E1 [29]. In the present study, we further revealed that the inhibiting effect of miR-15b on the proliferation and apoptosis of U87 and LN229 cell could 
be mediated by reducing the expression of Cyclin D1. Results from the luciferase reporter assay suggested that Cyclin D1 was one of the direct functional downstream targets of miR-15b. MiR-15b regulates Cyclin D1 expression through targeting the $3^{\prime}$ UTR of Cyclin D1, which was confirmed by western blotting analysis.

To conclude, our results show that miR-15b reduces in glioma specimens and cell lines, highlighting that miR-15b is a tumor suppressor miRNA in progression of glioma. Overexpression of miR-15b inhibits proliferation by arrested cell cycle progression and induces apoptosis, possibly by directly targeting Cyclin D1. Collectively, these data suggest that miR-15b and Cyclin D1 may be potential therapeutic targets for gliomagenesis and deserve further study.

\section{Conflict of Interests}

The authors declare that there is no conflict of interests regarding the publication of this paper. All authors have declared all sources of funding for the research reported in this paper and have no financial or other contractual agreements that might cause conflict of interests or be perceived as causing conflict of interests.

\section{Authors' Contribution}

Guan Sun, Lei Shi, and Shushan Yan contributed equally.

\section{Acknowledgments}

This work was supported by the China Natural Science Foundation (81201976, 81000963), Jiangsu Province's Natural Science Foundation (BK2012670), Jiangsu Province's Health Department (z201318), Yancheng Medical Science Development Foundation (YK2013003, YK2013019), Jiangsu Province's 333 Talent Program (BRA2011046), and the Kunshan Social Development Foundation (Grant nos. KS1006 and KS1009).

\section{References}

[1] H. Ohgaki and P. Kleihues, "Genetic alterations and signaling pathways in the evolution of gliomas," Cancer Science, vol. 100, no. 12, pp. 2235-2241, 2009.

[2] S. A. Grossman, X. Ye, S. Piantadosi et al., "Survival of patients with newly diagnosed glioblastoma treated with radiation and temozolomide in research studies in the United States," Clinical Cancer Research, vol. 16, no. 8, pp. 2443-2449, 2010.

[3] B. Auffinger, B. Thaci, A. Ahmed, I. Ulasov, and M. S. Lesniak, "MicroRNA targeting as a therapeutic strategy against Glioma," Current Molecular Medicine, vol. 13, no. 4, pp. 535-542, 2013.

[4] M. Karsy, E. Arslan, and F. Moy, "Current progress on understanding MicroRNAs in glioblastoma multiforme," Genes and Cancer, vol. 3, no. 1, pp. 3-15, 2012.

[5] B. Peng, D. Li, M. Qin et al., "MicroRNA218 inhibits glioma migration and invasion via inhibiting glioma-associated oncogene homolog 1 expression at $\mathrm{N}$ terminus," Tumor Biology, vol. 35, no. 4, pp. 3831-3837, 2014.
[6] L. Shi, Y. Wan, G. Sun et al., "Functional differences of miR$125 \mathrm{~b}$ on the invasion of primary glioblastoma CD133-negative cells and CD133-positive cells," NeuroMolecular Medicine, vol. 14, no. 4, pp. 303-316, 2012.

[7] G. Sun, Y. Cao, L. Shi et al., "Overexpressed miRNA-137 inhibits human glioma cells growth by targeting Racl," Cancer Biotherapy and Radiopharmaceuticals, vol. 28, no. 4, pp. 327334, 2013.

[8] X. R. Wang, H. Luo, H. L. Li et al., “Overexpressed let-7a inhibits glioma cell malignancy by directly targeting K-ras, independently of PTEN," Neuro-Oncology, vol. 15, no. 11, pp. 1491-1501, 2013.

[9] Y. Y. Wang, G. Sun, H. Luo et al., "Mir-21 modulates htert through a stat3-dependent manner on glioblastoma cell growth," CNS Neuroscience and Therapeutics, vol. 18, no. 9, pp. 722-728, 2012.

[10] J. Guo, B. Xia, F. Meng, and G. Lou, "miR-137 suppresses cell growth in ovarian cancer by targeting AEG-1," Biochemical and Biophysical Research Communications, vol. 441, no. 2, pp. 357363, 2013.

[11] P. Li, L. Ma, Y. Zhang, F. Ji, and F. Jin, "MicroRNA-137 down-regulates KIT and inhibits small cell lung cancer cell proliferation," Biomedicine and Pharmacotherapy, vol. 68, no. 1, pp. 7-12, 2013.

[12] X. Zhu, Y. Li, H. Shen et al., "miR-137 inhibits the proliferation of lung cancer cells by targeting Cdc42 and Cdk6," FEBS Letters, vol. 587, no. 1, pp. 73-81, 2013.

[13] W. G. Cao, R. Fan, L. F. Wang et al., "Expression and regulatory function of miRNA-34a in targeting survivin in gastric cancer cells," Tumor Biology, vol. 34, no. 2, pp. 963-971, 2013.

[14] L. Xia, D. Zhang, R. Du et al., "miR-15b and miR-16 modulate multidrug resistance by targeting BCL2 in human gastric cancer cells," International Journal of Cancer, vol. 123, no. 2, pp. 372379, 2008.

[15] C. S. Wu, C. J. Yen, R. H. Chou et al., "Downregulation of microRNA-15b by hepatitis B virus X enhances hepatocellular carcinoma proliferation via fucosyltransferase 2-induced Globo H expression," International Journal of Cancer, vol. 134, no. 7, pp. 1638-1647, 2014.

[16] Y. Xi, A. Formentini, M. Chien et al., "Prognostic values of microRNAs in colorectal cancer," Biomarker Insights, vol. 2, pp. 113-121, 2006.

[17] X. Wang, S. Tang, S. Y. Le et al., "Aberrant expression of oncogenic and tumor-suppressive microRNAs in cervical cancer is required for cancer cell growth," PLoS ONE, vol. 3, no. 7, Article ID e2557, 2008.

[18] G. E. Chung, J. H. Yoon, S. J. Myung et al., "High expression of microRNA-15b predicts a low risk of tumor recurrence following curative resection of hepatocellular carcinoma," Oncology Reports, vol. 23, no. 1, pp. 113-119, 2010.

[19] H. Xia, Y. Qi, S. S. Ng et al., "MicroRNA-15b regulates cell cycle progression by targeting cyclins in glioma cells," Biochemical and Biophysical Research Communications, vol. 380, no. 2, pp. 205-210, 2009.

[20] J. A. Diehl, "Cycling to cancer with cyclin D1," Cancer Biology and Therapy, vol. 1, no. 3, pp. 226-231, 2002.

[21] E. Tashiro, A. Tsuchiya, and M. Imoto, "Functions of cyclin D1 as an oncogene and regulation of cyclin D1 expression," Cancer Science, vol. 98, no. 5, pp. 629-635, 2007.

[22] X. Chen, T. Zhao, L. Li et al., "CCND1 G870A polymorphism with altered cyclin D1 transcripts expression is associated with 
the risk of glioma in a Chinese population," DNA and Cell Biology, vol. 31, no. 6, pp. 1107-1113, 2012.

[23] D. Dworakowska, "Clinical significance of cyclin D1 expression in non-small cell lung cancer," Pneumonologia i Alergologia Polska, vol. 73, no. 3, pp. 297-300, 2005.

[24] O. Gautschi, D. Ratschiller, M. Gugger, D. C. Betticher, and J. Heighway, "Cyclin D1 in non-small cell lung cancer: a key driver of malignant transformation," Lung Cancer, vol. 55, no. 1, pp. 114, 2007.

[25] Q. Yang, B. Wang, W. Gao et al., "SIRT1 is downregulated in gastric cancer and leads to G1-phase arrest via NF- $\kappa \mathrm{B} /$ cyclin D1 signaling," Molecular Cancer Research, vol. 11, no. 12, pp. 14971507, 2013.

[26] J. Wang, Q. Wang, Y. Cui et al., "Knockdown of cyclin D1 inhibits proliferation, induces apoptosis, and attenuates the invasive capacity of human glioblastoma cells," Journal of Neuro-Oncology, vol. 106, no. 3, pp. 473-484, 2012.

[27] L. Zheng, T. Qi, D. Yang et al., "microRNA-9 suppresses the proliferation, invasion and metastasis of gastric cancer cells through targeting cyclin D1 and Etsl," PLoS ONE, vol. 8, no. 1, Article ID e55719, 2013.

[28] Y. Y. Xu, H. J. Wu, H. D. Ma, L. Xu, Y. Huo, and L. Yin, "MicroRNA-503 suppresses proliferation and cell-cycle progression of endometrioid endometrial cancer by negatively regulating cyclin D1," The FEBS Journal, vol. 280, no. 16, pp. 3768-3779, 2013.

[29] W. Hui, L. Yuntao, L. Lun et al., "MicroRNA-195 inhibits the proliferation of human glioma cells by directly targeting cyclin D1 and cyclin E1," PLoS ONE, vol. 8, no. 1, Article ID e54932, 2013. 

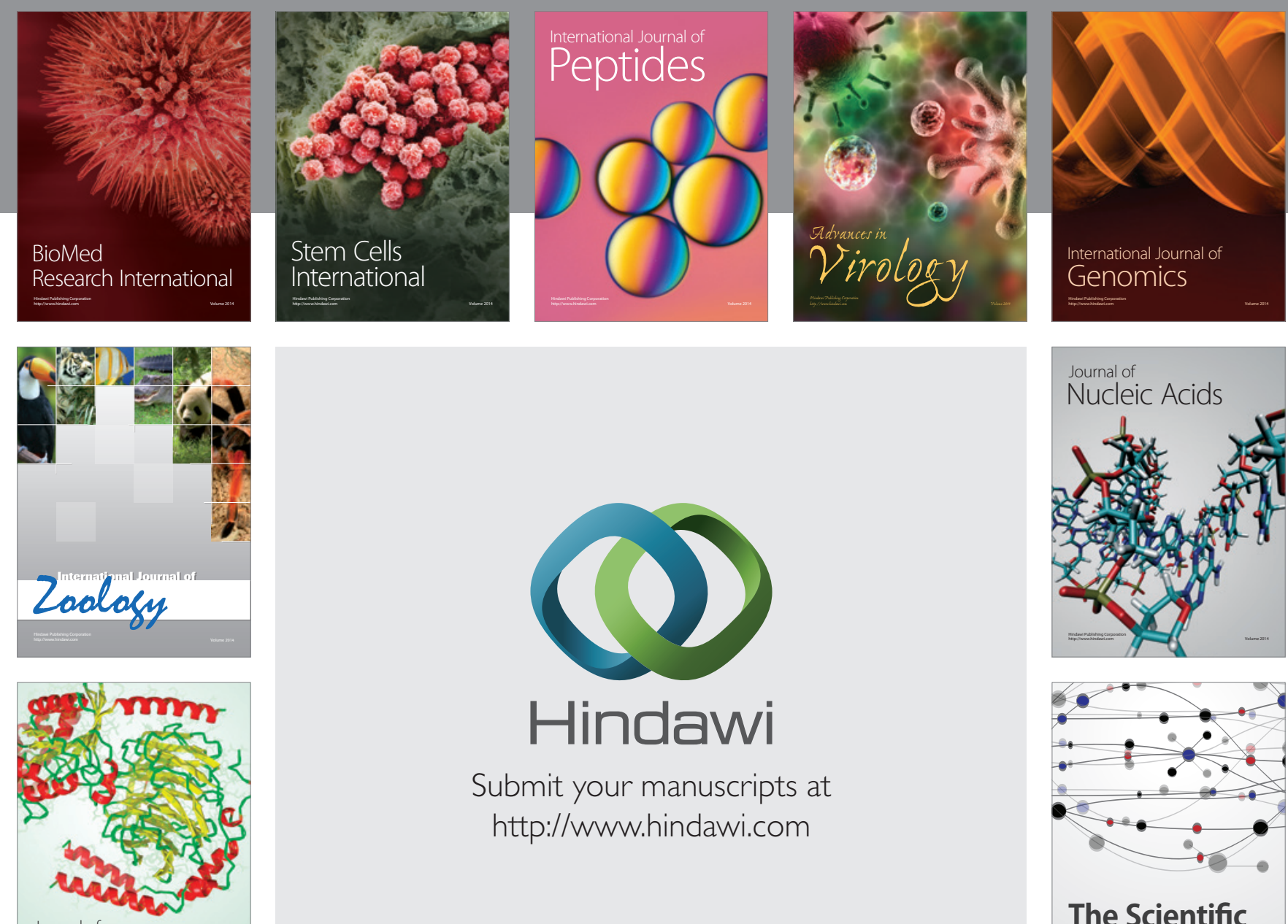

Submit your manuscripts at

http://www.hindawi.com

Journal of
Signal Transduction
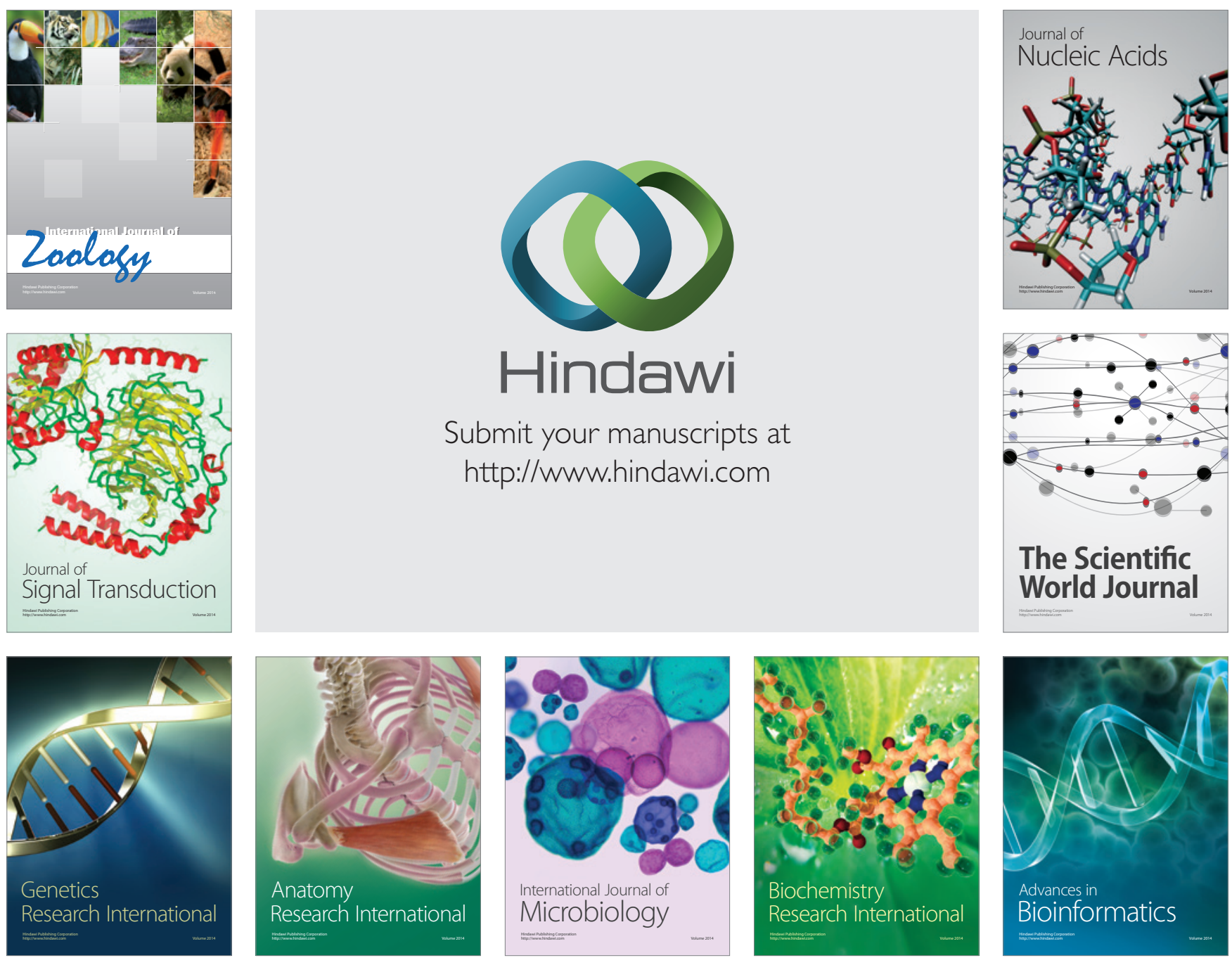

The Scientific World Journal
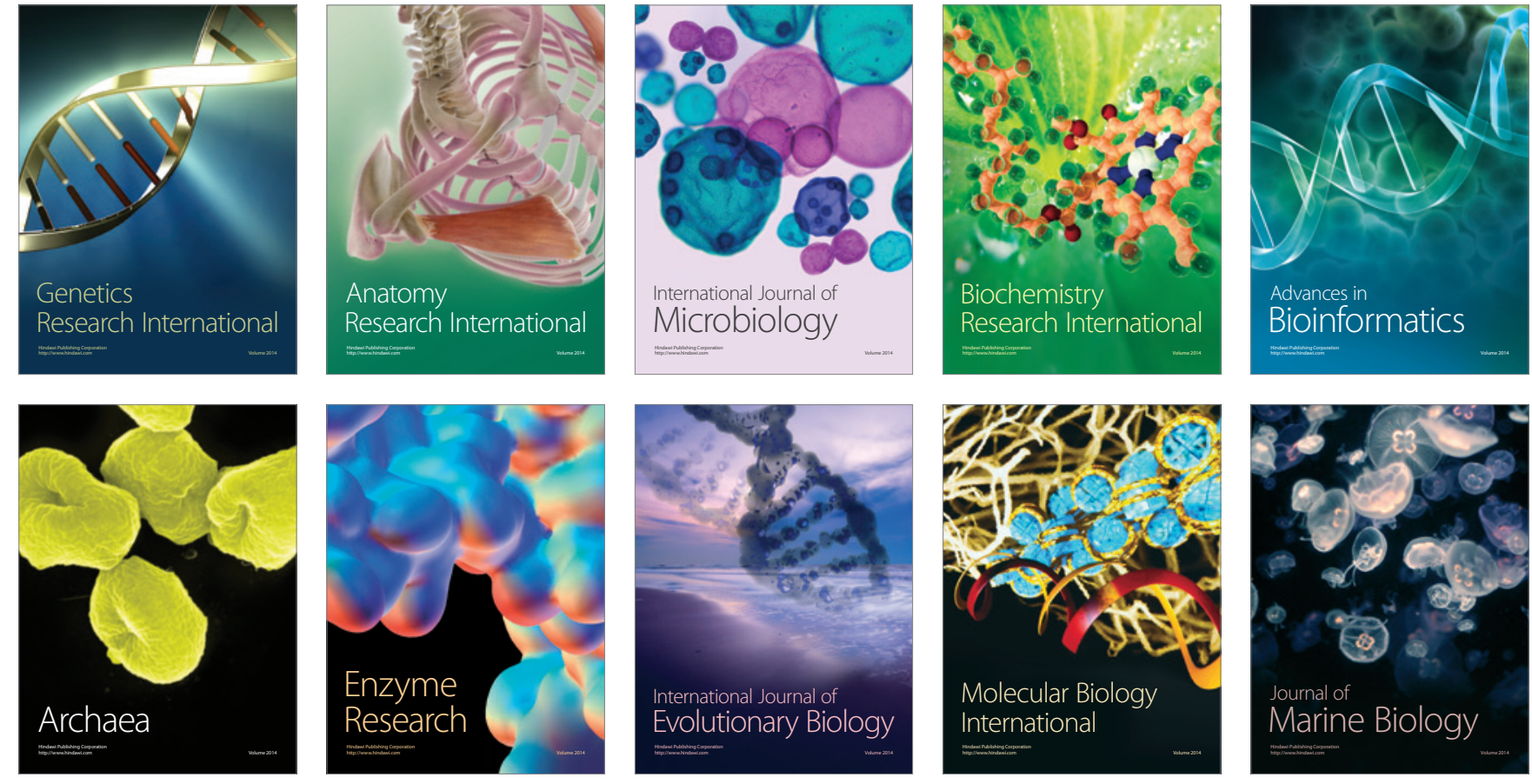